\title{
The Effect of Low-Dose Nitroglycerin Ointment on Skin Flap Necrosis in Breast Reconstruction after Skin-Sparing or Nipple-Sparing Mastectomy
}

\author{
Min Ho Yun, Eul Sik Yoon, Byung-Il Lee, Seung-Ha Park \\ Department of Plastic Surgery, Korea University Anam Hospital, Korea University College of Medicine, Seoul, Korea
}

Background Skin flap necrosis is a common complication after mastectomy and breast reconstruction. It has been proven that nitroglycerin ointment, as a topical vasodilator, can decrease the rate of skin flap necrosis after mastectomy and breast reconstruction. However, nitroglycerin can cause several side effects, including headache, dizziness, and hypotension. The purpose of this study was to evaluate whether the application of a low dose of nitroglycerin ointment reduced the rate of skin flap necrosis in breast reconstruction after skin-sparing or nipple-sparing mastectomy.

Methods A total of 73 cases of breast reconstruction after nipple-sparing and skin-sparing mastectomy at our institution from March 2012 to January 2017 were retrospectively studied. Of these patients, 52 received nitroglycerin ointment $(4.5 \mathrm{mg})$ application to the skin around the nipple-areolar complex from August 2015 to January 2017, while 21 received fusidic acid ointment from March 2012 to August 2015. The number of patients who experienced necrosis of the breast skin flap was counted in both groups.

Results Skin flap necrosis developed in $2(3.8 \%)$ patients who were treated with nitroglycerin ointment and $5(23.8 \%)$ patients who did not receive nitroglycerin ointment treatment. Patients who did not receive nitroglycerin ointment treatment had a significantly higher risk of mastectomy skin flap necrosis than patients who did (odds ratio $=7.81 ; 95 \%$ confidence interval, 1.38 to $44.23 ; \mathrm{P}=0.02$ ).

Conclusions Low-dose nitroglycerin ointment administration significantly decreased the rate of skin flap necrosis in patients who underwent breast reconstruction after skin-sparing or nipple-sparing mastectomy, without increasing the incidence of the side effects of nitroglycerin.

Keywords Nitroglycerin / Ointments / Skin / Flap / Necrosis
Correspondence: Eul Sik Yoon Department of Plastic Surgery, Korea University Anam Hospital, Korea University College of Medicine, 73 Inchon-ro, Seongbuk-gu, Seoul 02841 , Korea

Tel: +82-2-920-5368

Fax: $+82-2-922-7437$

E-mail:yesanam2@korea.ac.kr
This article was presented at the 2015 meeting of the Korean Society of Plastic and Reconstructive Surgeons on November 13-15, 2015 in Seoul, Korea.

Received: 2 Jun 2017 • Revised: 2 Aug 2017 • Accepted: 8 Sep 2017

pISSN: 2234-6163 • elSSN: 2234-6171 • https://doi.org/10.5999/aps.2017.00934 • Arch Plast Surg 2017;44:509-515

\section{INTRODUCTION}

Breast reconstruction after mastectomy continues to become more common, especially after the introduction of public health insurance coverage for breast reconstruction in Korea in 2015.

A variety of methods have been developed for breast reconstruction, ranging from autologous tissue transfer, including the latissimus dorsi flap and the free transverse rectus abdominis

Copyright $(\odot) 2017$ The Korean Society of Plastic and Reconstructive Surgeons

This is an Open Access article distributed under the terms of the Creative Commons Attribution Non-Commercial License (http://creativecommons.org/

licenses/by-nc/4.0/) which permits unrestricted non-commercial use, distribution, and reproduction in any medium, provided the original work is properly cited.

www.e-aps.org 
myocutaneous flap, to inserting a tissue expander or silicone implant. Nonetheless, mastectomy is performed prior to all such operations, and the viability of skin flaps after mastectomy is very important for the patient's therapeutic and cosmetic prognosis. During mastectomy, blood supply to the skin is compromised. This may lead to areas with mastectomy flap necrosis (Fig. 1) [1]. These areas may need additional surgical procedures, including surgical debridement and skin grafting. This may delay adjuvant treatment and diminish the cosmetic outcomes.

There are several reasons for vascular compromise and skin flap necrosis, but the main cause is thinned mastectomy skin flap. At the time of mastectomy, the breast surgeon performs surgery with the goal of completely removing the cancer. For this reason, breast surgeons tend to over-remove soft tissue in addition to the breast parenchyma.

Several studies have analyzed the relationship between the thickness of the remaining skin flap and the cancer recurrence rate after mastectomy to determine the optimal skin flap thickness in mastectomy $[2,3]$. However, the relationship between the thickness of the remaining skin flap and the cancer recurrence rate after mastectomy remains controversial. Therefore, skin flap thickness after mastectomy may vary depending on the preference of the breast surgeon.

Skin flap thickness plays an important role in skin flap survival. Arterial supply and venous drainage are accomplished through the subdermal plexus and underlying soft tissue. In thin flaps, the vascularity can be compromised and necrosis can occur. To overcome these problems, numerous agents have been studied with the goal of preventing flap necrosis and increasing the flap survival rate [4].

Nitroglycerin (NTG) ointment is not widely used for preventing skin flap necrosis, despite its potential for increasing skin flap survival, ease of application, and rapid onset time. The effects of topical NTG ointment on skin flap survival remain controversial. Several animal and clinical studies have reported negative effects of NTG ointment on skin flap survival. Hwang et al. [5] concluded that NTG ointment had no effect on skin flap survival. In a clinical study, Dunn et al. [6] reported that a single postoperative application of NTG was not more effective than a control ointment for preventing flap and graft complications.

However, a study by Rohrich et al. [7] showed that topical NTG could improve random flap survival in a rat model.

After the first study on NTG in human skin flaps was published by Scheuer and Hanna [8] in 1986, several clinical trials followed. Wong et al. [9] reported that topical NTG could increase the skin flap survival rate in humans. More recently, Gdalevitch et al. [1] reported that patients who received NTG ointment after mastectomy and immediate reconstruction showed a marked reduction in flap necrosis.

Several articles have reported the efficacy of NTG ointment $[1,10-13]$. However, both the doses and the dosing interval of the NTG ointment were different in these studies. Currently, there are no established guidelines for NTG ointment application.

In addition, NTG can cause side effects. Several studies have reported complications such as headache, dizziness, and hypotension, which can make patients stop using NTG ointment [10-15]. Moreover, these side effects are dose-dependent.

Therefore, we planned to reduce the dose of NTG ointment compared to the doses used in previous studies and determine whether NTG ointment could be effective at a low dose. The primary objective of this study was to evaluate whether a low dose of a NTG ointment (Rectogesic $4 \mathrm{mg} / \mathrm{g}$ [Kyowa Hakko Kirin Co., Ltd.], $4.5 \mathrm{mg}$ ) after primary breast reconstruction could decrease the rate of mastectomy flap necrosis in patients

Fig. 1. Skin flap necrosis after immediate breast reconstruction

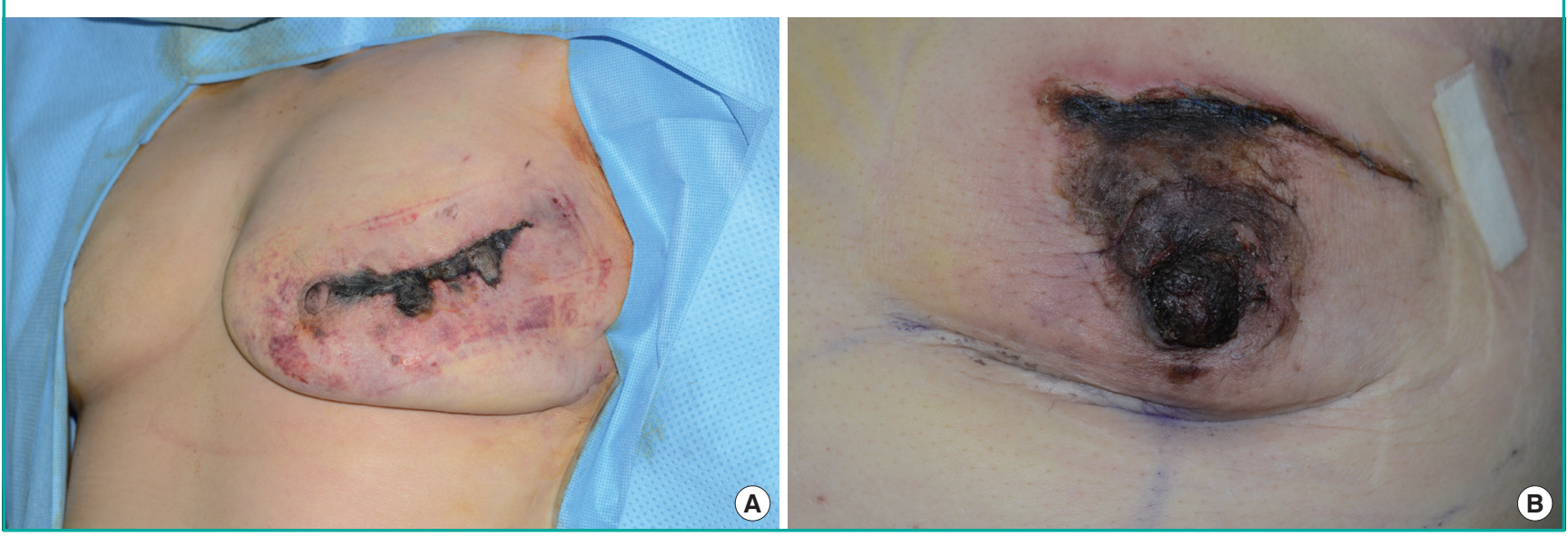


undergoing mastectomy with immediate breast reconstruction compared to patients receiving conventional fusidic acid ointment application.

\section{METHODS}

\section{Inclusion and exclusion criteria}

A total of 73 patients who underwent immediate breast reconstruction after mastectomy between March 2012 and January 2017 at our institution were retrospectively enrolled in this study. Starting in August 2015, NTG ointment was used at our institution. Therefore, patients who underwent breast reconstruction before August 2015 were analyzed as controls. Those who were treated after August 2015 were analyzed as the NTG group.

In order to reduce the bias from differences in surgical method, only cases of 2-stage alloplastic reconstruction using tissue expander placement were included, while cases of other types of autologous reconstruction, in which a latissimus dorsi flap or a transverse rectus abdominis flap was used, were excluded. Acellular dermal matrix was placed in all cases with tissue expander placement.

All reconstructions were performed by the senior author of this study. Mastectomies were performed by 2 different senior breast surgeons.

Patients with a medical history of conditions such as hypotension, heart or blood vessel disorders, closed-angle glaucoma, migraine or recurrent headaches, increased intracranial pressure (e.g., head trauma or cerebral hemorrhage) or inadequate cerebral circulation, and anemia, and patients who took medications such as sildenafil citrate, tadalafil, vardenafil, amyl, or butyl nitrite were excluded from this study for safety [16]. A medical chart review was performed retrospectively to collect the necessary data.

\section{Data collection}

The following information was collected: age; body mass index; smoking status; radiation exposure; chemotherapy exposure; medical comorbidities; breast size; dates and details of surgery, including the breast and plastic surgeon; type/size of alloplastic device and acellular dermal matrix; mastectomy incision; timing of drain removal; early complications such as infection, seroma, and hematoma; and the presence and details of skin flap necrosis (location, size, degree of skin flap necrosis, and treatment). Breast size was measured using the casting method with vinyl wrap and cotton plaster. In order to ensure accurate measurements with minimal bias, breast volume was measured by a single nurse in our clinic in all cases.

\section{Surgical and dressing method}

Mastectomy was performed by 2 senior breast surgeons who utilized electrocautery followed by immediate reconstruction by a single senior plastic surgeon.

All mastectomies were done through periareolar and lateral extension incisions.

In all cases, dental lidocaine diluted with saline at a 1:3 ratio was used. A 2-stage procedure consisting of tissue expander insertion followed by permanent silicone implant was employed.

All reconstructions were performed using the standard submuscular approach while minimizing dissection of the medial fibers of the pectoralis muscle. For each operation, the tissue expander or permanent implant was completely covered with muscle and acellular dermal matrix.

All tissue expanders began to be inflated 2 weeks after the operation. Thereafter, expansion of the tissue expander was continued at intervals of 2 weeks until the target volume was reached.

All cases were drained with 10-Fr Barovac drains (Sewoon Medical, Cheonan, Korea) either until the consecutive 24-hour output was less than $30 \mathrm{~mL}$ or until 2 weeks postoperatively.

All patients received intravenous antibiotics 30 minutes before the surgical incision, along with a 7-day course of intravenous antibiotics postoperatively.

All patients received the same postoperative dressing, except for the type of ointment used. In the NTG group, at the time of dressing application after surgery, the senior surgeon (E.S.Y.) applied NTG ointment from the center of the breast to the periphery at a dose of $4.5 \mathrm{mg}$ of NTG ointment (equivalent to 7.5 $\mathrm{cm}$ on the measuring strip) (Fig. 2). Each patient was treated with individually unpacked NTG ointment to reduce the risk of infection or contamination. The patient's blood pressure was monitored in the immediate postoperative setting. Antibacterial petrolatum gauze was then used to cover the incision site. Finally, 2 rolls of 6-inch elastic bandage were wrapped around the patient's chest and back for mild compression to prevent hematoma formation. All patients received dressing changes every other day as described above. NTG ointment was applied 3 times (on postoperative days 2, 4, and 6). After that, antibacterial fusidic acid ointment was used instead of the NTG ointment. At 2 weeks after surgery, all stitches were taken out. No dressing was needed. In the control group, all dressing procedures were the same as those used for the NTG group, except for the type of ointment. Antibacterial fusidic acid ointment was applied to all patients in the control group.

Patients with areas of skin necrosis continued to receive dressing changes every other day with NTG or fusidic acid ointment until the ischemic skin change resolved or the necrotic skin was 


\section{Fig. 2. Application of nitroglycerin ointment}

(A) When the dressing was applied after surgery, $7.5 \mathrm{~cm}$ of nitroglycerin ointment, which was equivalent to $4.5 \mathrm{mg}$ of nitroglycerin, was prepared with a ruler. (B) The senior surgeon applied the nitroglycerin ointment from the center of the breast to the periphery.



demarcated.

If the ischemic change did not heal spontaneously, patients with skin necrosis underwent surgical debridement after the lesion was completely demarcated.

\section{Statistical analysis}

In order to show the efficacy of NTG ointment on mastectomy skin flap survival and to exclude other factors, 10 independent variables known to be risk factors for skin flap necrosis were analyzed together: age, body mass index, smoking, diabetes mellitus, hypertension, previous chemotherapy, previous radiotherapy, the breast surgeon, the reconstruction modality, and breast volume.

To obtain a final model, binary logistic regression was performed. Backward selection was then performed based on Wald statistics to remove non-significant terms from the model. All results are presented as odds ratios (ORs) with 95\% confidence intervals (CIs). The Pearson chi-square test was used to compare the proportions of side effects between the 2 groups. P-values $<0.05$ were considered to indicate statistical significance. All data were analyzed using SPSS ver. 20.0 for Windows (IBM Corp., Armonk, NY, USA).

\section{RESULTS}

\section{Patient characteristics}

A total of 73 patients were included in this study, including 21 in the control group and 52 in the NTG ointment group.

No statistically significant differences in patient demographics were found between the 2 groups (Table 1 ). The mean ages of the control group and the NTG group were 45.0 and 47.0 years,

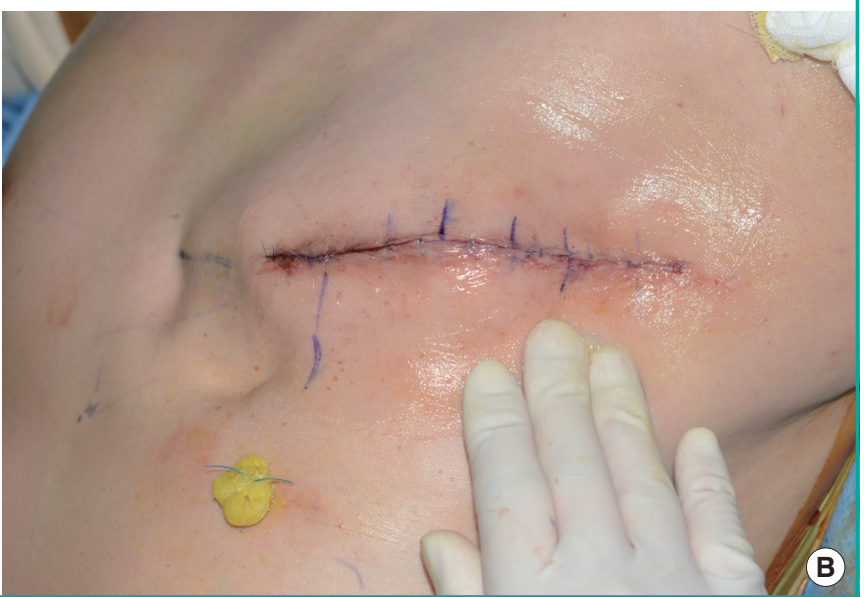

Table 1. Patient demographics in the control and nitroglycerin ointment groups

\begin{tabular}{|lcc|}
\hline Demographics & $\begin{array}{c}\text { Control group } \\
(\mathbf{n}=\mathbf{2 1})\end{array}$ & $\begin{array}{c}\text { NTG group } \\
(\mathbf{n}=\mathbf{5 2})\end{array}$ \\
\hline Age $(\mathrm{yr})$ & & \\
$\quad$ Mean $\pm \mathrm{SD}$ & $45.0 \pm 8.1$ & $47.0 \pm 9.6$ \\
$\quad$ Median & 44.0 & 46.0 \\
BMl $\left(\mathrm{kg} / \mathrm{m}^{2}\right)$ & & \\
$\quad$ Mean $\pm \mathrm{SD}$ & $21.0 \pm 2.3$ & $24.0 \pm 3.7$ \\
$\quad$ Median & 20.7 & 23.0 \\
Smoking history (\%) & $2(9.5)$ & $1(1.9)$ \\
Diabetes (\%) & 0 & $3(5.8)$ \\
Hypertension (\%) & $1(4.8)$ & $3(5.8)$ \\
Preoperative chemotherapy & 0 & $2(3.8)$ \\
Preoperative radiotherapy & 0 & 0 \\
\hline Values are presented as mean \pm standard deviation (SD) or number (\%). \\
NTG, nitroglycerin; BMl, body mass index. & \\
\hline
\end{tabular}

respectively. The mean body mass index of the control group was $21.0 \mathrm{~kg} / \mathrm{m}^{2}$, and that of the NTG group was $24.0 \mathrm{~kg} / \mathrm{m}^{2}$. Two (9.5\%) patients in the control group and 1 (1.9\%) in the NTG group were current smokers. No (0\%) patients in the control group and 3 (5.8\%) in the NTG group had diabetes mellitus. One (4.8\%) patient in the control group and $3(5.8 \%)$ in the NTG group had hypertension. Preoperative chemotherapy was administered to no (0\%) patients in the control group and $2(3.8 \%)$ patients in the NTG group. There were no cases of preoperative radiation therapy in either group.

The breast size of patients was grouped according to $100-\mathrm{mL}$ units. Breast size showed a tendency to be larger in the NTG group than in the control group. In both groups, the percentage of patients who underwent mastectomy by surgeon A was slightly higher than the percentage of patients operated on by 
Table 2. Surgical factors in the control and nitroglycerin ointment groups

\begin{tabular}{|lcc|}
\hline Variable & $\begin{array}{c}\text { Control group } \\
(\mathbf{n}=\mathbf{2 1})\end{array}$ & $\begin{array}{c}\text { Nitroglycerin group } \\
(\mathbf{n}=\mathbf{5 2})\end{array}$ \\
\hline $\begin{array}{l}\text { Breast size }(\mathrm{mL}) \\
<200\end{array}$ & $6(28.6)$ & $12(23.1)$ \\
$201-300$ & $5(23.8)$ & $26(50.0)$ \\
$301-400$ & $9(42.9)$ & $9(17.3)$ \\
$400<$ & $1(4.8)$ & $5(9.6)$ \\
Breast surgeon & & \\
A & $12(57.1)$ & $29(55.8)$ \\
B & $9(42.9)$ & $23(44.2)$ \\
\hline
\end{tabular}

Table 3. Factors associated with skin flap necrosis in the final model

\begin{tabular}{|lcccc|}
\hline \multirow{2}{*}{ Varaible } & \multirow{2}{*}{$\begin{array}{l}\text { Odds } \\
\text { ratio }\end{array}$} & \multicolumn{2}{c}{$\begin{array}{c}95 \% \text { Confidence interval } \\
\text { for odds ratio }\end{array}$} & P-value \\
\cline { 3 - 4 } & & Lower & Upper & \\
\hline NTG ointment (No) & 7.81 & 1.38 & 44.23 & 0.02 \\
\hline
\end{tabular}

This was obtained from the model including the covariates such as age, BMI, smoking, DM, HTN, previous chemotherapy, previous radiotherapy, breast surgeon, reconstruction modality, breast size and NTG ointment application using the backward variable selection.

NTG, nitroglycerin; BMI, body mass index; DM, diabetes mellitus; HTN, hypertension.

surgeon B (Table 2).

\section{Risk of skin flap necrosis}

Binary logistic regression was performed to eliminate covariates in the final model.

The final model of logistic regression analysis (Table 3) demonstrated that application of the NTG ointment was significantly and independently associated with mastectomy skin flap necrosis. The control group had a significantly higher risk ( $\mathrm{OR}=$ 7.81; 95\% CI, 1.38-44.23; P = 0.02) of mastectomy skin flap necrosis than the NTG group.

Full-thickness skin flap necrosis was only observed in the control group, in which there were 3 cases of full-thickness necrosis and 2 of superficial-thickness necrosis. The 3 patients with fullthickness skin necrosis required surgical debridement and delayed repair after demarcation of the wound. There were 2 cases of skin flap necrosis in the NTG group, all of which were superficial-thickness necrosis.

In both groups, the ischemic skin changes in cases of superficial-thickness skin flap necrosis were resolved by dressing changes every other day with ointment.

Explantation of an alloplastic device was not necessary in any skin flap necrosis cases (Table 4).
Table 4. Post-mastectomy flap necrosis in the control and nitroglycerin groups

\begin{tabular}{|lcc|}
\hline Varaible & Control (\%) & Nitroglycerin (\%) \\
\hline Skin flap necrosis & $5(23.8)$ & $2(3.8)$ \\
Superficial thickness & $2(9.5)$ & $2(3.8)$ \\
Full thickness & $3(14.3)$ & 0 \\
Surgical debridement & $3(14.3)$ & 0 \\
Explantation & 0 & 0 \\
\hline
\end{tabular}

Table 5. Complication rate in the control and nitroglycerin groups

\begin{tabular}{|lccc|}
\hline Varaible & Control (\%) & Nitroglycerin (\%) & P-value \\
\hline Headache & $2(9.5)$ & $11(21.2)$ & 0.24 \\
Dizziness & $3(14.3)$ & $5(9.6)$ & 0.56 \\
Hypotension & 0 & 0 & \\
\hline
\end{tabular}

\section{Side effects}

Common side effects of NTG ointment application are headache, dizziness, and hypotension [10-15]. In our study, no hypotension was reported in either group. Headache and dizziness were likewise not correlated with NTG ointment application (Table 5). Two (9.5\%) of the 21 patients of the control group and $11(21.2 \%)$ of the 52 patients in the NTG group experienced headache $(\mathrm{P}=0.24)$. Three $(14.3 \%)$ patients in the control group and 5 (9.6\%) patients in the NTG group experienced dizziness. There was no statistically significant difference between the 2 groups in terms of dizziness $(\mathrm{P}=0.56)$.

\section{DISCUSSION}

Skin flap necrosis is a relatively common complication after mastectomy. It occurs in $2.5 \%$ to $60 \%$ of all patients. The rate varies greatly depending on the breast and reconstructive surgeon, patient selection, surgical technique, and other factors [17-20]. Different agents have been used to improve the survival of flaps. In this study, the effect of NTG ointment at a low dose was studied.

It is known that topical NTG can induce smooth muscle relaxation in vessel walls, resulting in venous and arterial vessel dilation [21]. Topical NTG can also cause endothelial cells to synthesize prostacyclin, which can inhibit platelet activation. Prostacyclin is an effective vasodilator, which may contribute to flap survival by decreasing the thrombosis of smaller vessels [22].

Dent et al. [23] reported that the patient factors associated with higher rates of nipple-areolar complex ischemia were similar to those associated with a high skin necrosis rate. In their study, older age, higher body mass index, greater breast volume, 
and a history of diabetes were found to have negative effect on flap survival. Thus, we collected data regarding these factors and performed binary logistic regression to exclude the effect of such variables in our evaluation of the effect of NTG ointment on the flap survival rate.

The objective of our study was to determine whether low-dose application of NTG ointment contributed to skin flap survival without causing side effects. Therefore, we reviewed articles in attempt to find the optimal dose and dosing interval of NTG without causing side effects.

Dunn et al. [6] showed that application of an NTG ointment on skin flaps or grafts was not more effective than the control ointment. In their study, the NTG ointment was applied at a dose of $6 \mathrm{mg}$ just once as part of the immediate postoperative dressing [10]. However, Zhuang et al. [24] observed a gradual increase of compensatory skin flap blood perfusion and a dilated vascular network over the course of 7 days after flap elevation in a rat model. Furthermore, a single application of NTG ointment is known to be effective for up to 12 hours [10]. Thus, we hypothesized that more frequent applications, instead of a single application, would be necessary to aid flap survival.

Gdalevitch et al. [1] reported promising results regarding the flap survival rate after NTG ointment application. In their study, patients were given a single application of NTG ointment with a dose of $45 \mathrm{mg}$. However, severe headache or hypotension occurred in several patients.

Many studies have proved that even low doses of NTG can cause frequent side effects including hypotension, flushing, and dizziness, suggesting that NTG is not well tolerated by some patients [10-15]. Dalsgaard-Nielsen [25] described a topical percutaneous NTG test, in which migraine attacks were provoked by an NTG ointment that was applied to the forehead and the temporal region. The threshold for headache was $6 \mathrm{mg}$ for healthy patients and $2 \mathrm{mg}$ for migraine patients. Hyman and Cataldo [13] reported that 29 of 33 patients experienced adverse reactions (specifically, headaches and light-headedness) after the application of a small amount of $0.3 \%$ NTG ointment (with $1 \mathrm{~cm}$ equivalent to $1 \mathrm{mg}$ of NTG) into their anoderm. Coperchini and Kreeger [11] suggested that the application of a small amount of $0.2 \%$ NTG ointment (with $1 \mathrm{~cm}$ equivalent to $0.75 \mathrm{mg}$ of NTG) onto the anus could cause postural hypotension and dizziness. Based on these results, we determined the dose of NTG ointment and the interval of application to be 4.5 mg once every 2 days, which was lower than the threshold dose for headache with a longer interval than the NTG ointment action time presented above. We investigated whether such a dose and interval were effective for flap survival.

Applying 4.5 mg of NTG ointment once every 2 days signifi- cantly reduced the flap necrosis rate. The control group had a risk of mastectomy skin flap necrosis that was more than 6times higher than the risk in the NTG group. Nevertheless, side effects in the NTG group were not significantly more common than in the control group.

This study has several limitations. First, it was a retrospective study. Second, we selected an NTG ointment dose of $4.5 \mathrm{mg}$ after reviewing previously published articles on the effects of topical NTG ointment on skin flaps. However, it is possible that NTG could also be effective for skin flap survival at even lower doses and longer intervals. Therefore, a further prospective study is necessary to investigate the application of various NTG ointment doses on patients and to compare the skin necrosis rates among various dose groups.

Furthermore, flap necrosis is closely associated with the breast surgeon's surgical skill and experience. In our study, we retrospectively analyzed patient data from a period of 6 years. Therefore, it is possible that the accumulation of technical experience by the breast surgeon during that period influenced the results of this study. However, the breast surgeons in this study were senior surgeons who had already passed the learning curve. The accumulation of technical experience by the breast surgeons during the study likely had a limited impact on the outcomes of this study.

Despite these limitations, our study provided evidence that the low-dose application of NTG ointment might be beneficial for skin flap survival after immediate breast reconstruction.

As outlined previously, despite its promising anti-skin flap necrosis effect, NTG ointment does have important side effects that merit consideration.

Using an appropriate dose and dosing interval when applying NTG ointment is crucial for patient safety. Thorough patient monitoring after administration of this agent is also essential.

\section{CONFLICT OF INTEREST}

No potential conflict of interest relevant to this article was reported.

\section{PATIENT CONSENT}

The patient provided written informed consent for the publication and the use of their images.

\section{REFERENCES}

1. Gdalevitch P, Van Laeken N, Bahng S, et al. Effects of nitroglycerin ointment on mastectomy flap necrosis in immedi- 
ate breast reconstruction: a randomized controlled trial. Plast Reconstr Surg 2015;135:1530-9.

2. Larson DL, Basir Z, Bruce T. Is oncologic safety compatible with a predictably viable mastectomy skin flap? Plast Reconstr Surg 2011;127:27-33.

3. Robertson SA, Rusby JE, Cutress RI. Determinants of optimal mastectomy skin flap thickness. Br J Surg 2014;101: 899-911.

4. Berner B, John VA. Pharmacokinetic characterisation of transdermal delivery systems. Clin Pharmacokinet 1994; 26:121-34.

5. Hwang CJ, Morgan PV, Pimentel A, et al. Rethinking the role of nitroglycerin ointment in ischemic vascular filler complications: an animal model with ICG imaging. Ophthal Plast Reconstr Surg 2016;32:118-22.

6. Dunn CL, Brodland DG, Griego RD, et al. A single postoperative application of nitroglycerin ointment does not increase survival of cutaneous flaps and grafts. Dermatol Surg 2000;26:425-7.

7. Rohrich RJ, Cherry GW, Spira M. Enhancement of skin-flap survival using nitroglycerin ointment. Plast Reconstr Surg 1984;73:943-8.

8. Scheuer S, Hanna MK. Effect of nitroglycerin ointment on penile skin flap survival in hypospadias repair. Experimental and clinical studies. Urology 1986;27:438-40.

9. Wong AF, McCulloch LM, Sola A. Treatment of peripheral tissue ischemia with topical nitroglycerin ointment in neonates. J Pediatr 1992;121:980-3.

10. Berry SM, Barish CF, Bhandari R, et al. Nitroglycerin $0.4 \%$ ointment vs placebo in the treatment of pain resulting from chronic anal fissure: a randomized, double-blind, placebocontrolled study. BMC Gastroenterol 2013;13:106.

11. Coperchini ML, Kreeger LC. Postural hypotension from topical glyceryl trinitrate ointment for anal pain. J Pain Symptom Manage 1997;14:263-4.

12. Gorfine SR. Topical nitroglycerin therapy for anal fissures and ulcers. N Engl J Med 1995;333:1156-7.

13. Hyman NH, Cataldo PA. Nitroglycerin ointment for anal fissures: effective treatment or just a headache? Dis Colon Rectum 1999;42:383-5.

14. Siddiqi M, Marco AP, Gorp CV. Postoperative hypotension from topical use of $2 \%$ nitroglycerin ointment after a total knee replacement procedure. J Clin Anesth 2004;16:77-8.

15. Tfelt-Hansen PC, Tfelt-Hansen J. Nitroglycerin headache and nitroglycerin-induced primary headaches from 1846 and onwards: a historical overview and an update. Headache 2009;49:445-56.

16. Kyowa Kirin Ltd. Rectogesic $4 \mathrm{mg} / \mathrm{g}$ rectal ointment [Internet]. Dublin: Kyowa Kirin Ltd; 2017 [cited 2017 Oct 11]. Available from: http://www.medicines.ie/history/16645/ SPC/Rectogesic+4+mg+g+Rectal+Ointment.

17. Komorowski AL, Zanini V, Regolo L, et al. Necrotic complications after nipple- and areola-sparing mastectomy. World J Surg 2006;30:1410-3.

18. Regolo L, Ballardini B, Gallarotti E, et al. Nipple sparing mastectomy: an innovative skin incision for an alternative approach. Breast 2008;17:8-11.

19. Garwood ER, Moore D, Ewing C, et al. Total skin-sparing mastectomy: complications and local recurrence rates in 2 cohorts of patients. Ann Surg 2009;249:26-32.

20. Algaithy ZK, Petit JY, Lohsiriwat V, et al. Nipple sparing mastectomy: can we predict the factors predisposing to necrosis? Eur J Surg Oncol 2012;38:125-9.

21. Needleman P, Corr PB, Johnson EM Jr. Drugs used for the treatment of angina: organic nitrates, calcium channel blockers, and beta-adrenergic antagonists. In: Gilman AG, editor. The pharmacological basis for therapeutics. 7 th ed. New York: Macmillan; 1985. p.806-26.

22. Levin RI, Jaffe EA, Weksler BB, et al. Nitroglycerin stimulates synthesis of prostacyclin by cultured human endothelial cells. J Clin Invest 1981;67:762-9.

23. Dent BL, Small K, Swistel A, et al. Nipple-areolar complex ischemia after nipple-sparing mastectomy with immediate implant-based reconstruction: risk factors and the success of conservative treatment. Aesthet Surg J 2014;34:560-70.

24. Zhuang Y, Xie Y, Wu Z, et al. Hemodynamic study of three patterns of flaps on rats with a novel panoramic photographing technique involved. Plast Reconstr Surg 2016;138:653e665 e.

25. Dalsgaard-Nielsen T. Migraine diagnostics with special reference to pharmacological tests. Int Arch Allergy Appl Immunol 1955;7:312-22. 